\title{
Silencing of NOD2 protects against diabetic cardiomyopathy in a murine diabetes model
}

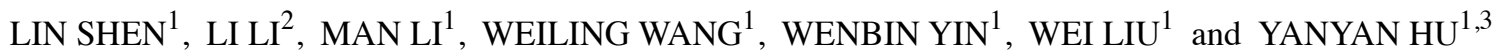 \\ ${ }^{1}$ Department of Geriatrics, Shandong Qilu Hospital; ${ }^{2}$ The Undergraduate Teaching Department, \\ Shandong University Qilu Medical College; ${ }^{3}$ Shandong Provincial Key Laboratory of \\ Cardiovascular Proteomics, Shandong University, Jinan, Shandong 250012, P.R. China
}

Received February 6, 2018; Accepted July 24, 2018

DOI: $10.3892 /$ ijmm.2018.3880

\begin{abstract}
The aim of the present study was to investigate the role of the nucleotide-binding oligomerization domain (NOD) 2 in high glucose (HG)-induced myocardial apoptosis and fibrosis in mice. Mouse models of diabetes were induced by streptozotocin (STZ). NOD2 expression was knocked down by injection of lentivirus-mediated short-hairpin RNA. Alternatively, small interfering RNA-NOD2 was transfected into cardiomyocytes and cardiac fibroblasts (CFs). A hemodynamic assay was used to assess the cardiac function in the mouse model. Hematoxylin and eosin, Masson and terminal deoxynucleotidyl transferase dUTP nick end labeling staining was performed to observe pathological changes and injury of myocardial tissue. The expression levels of NOD2, collagen I and III, and transforming growth factor- $\beta(\mathrm{TGF}-\beta)$ and apoptotic proteins were quantified by reverse transcription-quantitative polymerase chain reaction and western blotting. NOD2 silencing ameliorated diabetes-induced myocardial apoptosis and fibrosis in mice. NOD2, collagen I, collagen III, TGF- $\beta$ and pro-apoptotic proteins were upregulated in the diabetic cardiomyopathy (DCM) model group, but interference of NOD2 suppressed these alterations in protein expression levels. NOD2 is upregulated in HG-induced primary cardiomyocytes and CFs. Suppression of NOD2 attenuated HG-induced cardiomyocyte apoptosis and proliferation of CFs. Overall, NOD2 silencing alleviated myocardial apoptosis and fibrosis in diabetic mice. The results of the present study demonstrated an understanding of the role of NOD2 in diabetes-induced cardiomyopathy, which provides a novel target and therapies for the prevention and treatment of DCM.
\end{abstract}

Correspondence to: Dr Yanyan Hu, Department of Geriatrics, Shandong Qilu Hospital, 107 Wen Hua West Road, Jinan, Shandong 250012, P.R. China

E-mail: huyanyan26@163.com

Key words: nucleotide-binding oligomerization domain, diabetic cardiomyopathy, high glucose, apoptosis, fibrosis

\section{Introduction}

Diabetes mellitus is associated with increased risk of heart attack, called diabetic cardiomyopathy (DCM). This condition is one of the leading causes of mortality with common cardiovascular complications (1). DCM alters the diastolic and systolic cardiac functions, resulting in myocardial ischemia and heart failure $(2,3)$. It is characterized by myocardial dysfunction, myocardial cell apoptosis and myocardial fibrosis, resulting from blood glucose metabolic abnormalities $(4,5)$. It is reported that high glucose (HG) can induce the expression of inflammatory cytokines and promote myocardial cell apoptosis, suggesting that cell apoptosis is closely associated with cardiovascular disease (6). Accumulating evidence has implicated oxidative stress and inflammation induced-apoptosis as contributors to the onset and development of DCM (7-9).

Cardiomyocyte apoptosis is a main factor in the pathology of DCM, which causes the loss of myocardial contractile cells, aggravates myocardial injury, accelerates the occurrence of cardiac dysfunction and ultimately may lead to heart failure $(10,11)$. Another important pathological feature of DCM is hyperglycemia-induced excess production of the extracellular matrix (ECM), including collagen types I and III, which are involved in cardiac remodeling and fibrosis (12). In vitro, transforming growth factor- $\beta$ (TGF- $\beta 1$ ) plays a critical role in alteration of the cardiac structure and function by differentiation of cardiac fibroblasts (CFs) and ECM deposition (13). In particular, activation and proliferation of CFs plays an important role in myocardial fibrosis (14). Hyperglycemia induces inflammation-associated cytokine expression, such as interleukin (IL)-1, tumor necrosis factor- $\alpha$ (TNF- $\alpha$ ) and TGF- $\beta 1$, which participate in the development and progression of myocardial fibrosis $(15,16)$.

Nucleotide oligomerization domain (NOD)-like receptors are a newly discovered set of pattern-recognition receptors. NOD2 as one of the members of the NOD-like receptor family have been demonstrated to activate transcription factors by mediating production of inflammatory cytokines $(17,18)$. The association of NOD2 with the mechanism of diabetic nephropathy, atherosclerosis, myocardial reperfusion injury and diabetes mellitus has been revealed in clinical and animal models (19-23). However, little is known about the role of NOD2-mediated apoptosis and fibrosis in DCM. 
The present study focused on the underlying mechanism of NOD2 in DCM, and demonstrated the effect of NOD2 silencing on HG-induced apoptosis and fibrosis, as well as on apoptosis and fibrosis-related proteins in a DCM model of mice. To further investigate the association between NOD2 and apoptosis or fibrosis-related proteins in cardiomyocytes and fibroblasts, NOD2 knockdown experiments were performed.

\section{Materials and methods}

Animal experiments and RNA interference. 8-9 week-old male C57BL/6 mice ( $\mathrm{n}=40$ ), (weighing 22-35 g) and 7-8 week-old timed pregnant C57BL/6 mice $(n=10$, mated with syngeneic males) were purchased from Experimental Animal Center of Shandong University (Jinan, China). Mice were maintained at a temperature of $22^{\circ} \mathrm{C}$ and a $55 \%$ humidity controlled environment on a $12 \mathrm{~h}$ light-dark cycle with free access to food and water. All experimental protocols were approved by the Institutional Animal Care and Use Committee of Shandong University. All surgeries were performed under sodium pentobarbital anesthesia.

The diabetes model was established by intraperitoneal injection of STZ (Sigma Aldrich; Merck KGaA, Darmstadt, Germany) dissolved in $0.1 \mathrm{ml}$ citrate buffer ( $\mathrm{pH} 4.5)$ intraperitoneally (i.p.) at $90 \mathrm{mg} / \mathrm{kg}$ per mouse for 6 consecutive days. Control mice were injected using equal parts of citrate buffer. After 1 week, the blood was obtained from the tail vein, and random glucose levels were measured by glucometer (Accu-chek Advantage; Roche Diagnostics GmbH, Mannheim, Germany). Diabetes was determined as blood glucose $>16.7 \mathrm{mmol} / 1$ (normally $11.1 \mathrm{mmol} / \mathrm{l}$ ). Following the induction of diabetes for 12 weeks, mice were randomly divided into 4 groups: Control $(n=8)$; diabetes model $(n=8)$; NOD2 short hairpin (sh)RNA mice $(\mathrm{n}=8)$ injected with $1 \times 10^{7} \mathrm{UT} / 30 \mu \mathrm{l}$ pLV-NOD2 shRNA (Shanghai Jikai Biological Technology Co., Ltd., Shanghai, China) in various sites of the left ventricle; shRNA negative control $(\mathrm{NC})(\mathrm{n}=8)$, mice were injected with $1 \times 10^{7} \mathrm{UT} / 30 \mu 1$ lenti-vehicle in various sites of the left ventricle. 293 T cells were used as an interim cell line to generate the packaged lentivirus. After $72 \mathrm{~h}$, levels of NOD2 expression were detected in tissues to assess efficiency of RNA interference in mice. Then, after 4 weeks, clinical examination was performed in these groups, and mice were sacrificed to obtain mouse blood and heart tissue for the following experiments.

Cell culture and treatment. The primary cardiomyocytes and primary CFs were isolated from the ventricular tissues of neonatal mice ( $<3 \mathrm{~d}$ old) from pregnant C57BL/6 mice. Cells were seeded and incubated in Dulbecco's modified Eagle's medium (DMEM; Gibco; Thermo Fisher Scientific, Inc., Waltham, MA, USA) supplemented with $10 \%$ fetal bovine serum (FBS; Gibco; Thermo Fisher Scientific, Inc.), 2 mM glutamine (Shanghai Biological Engineering Co., Ltd., Shanghai, China) and antibiotics (100 U/ml penicillin and $100 \mathrm{ug} / \mathrm{ml}$ streptomycin; Gibco; Thermo Fisher Scientific, Inc.), incubated at $37^{\circ} \mathrm{C}$ in a humidified incubator containing $5 \% \mathrm{CO}_{2}$ and $95 \%$ air. Cells were identified using $\alpha$-sarcomeric actin and FSP-1 stain to confirm that $>90 \%$ of cells were cardiomyocytes and CFs, respectively. Cultured cell populations reached $60 \%$ confluence, and were treated in the presence or absence of HG (15-25 $\mathrm{mM})$ for $48 \mathrm{~h}$.

Immunofluorescence. Immunofluorescent staining was performed using cultured primary cells. A total of $2.0 \times 10^{4}$ neonatal cardiomyocytes and CFs were seeded on glass coverslips $\left(2 \mathrm{~cm}^{2}\right)$ wells, cultured for $24 \mathrm{~h}$ in DMEM with $10 \%$ FBS and washed with PBS, then fixed using $4 \%$ paraformaldehyde for $20 \mathrm{~min}$ at room temperature. Cells were then washed 3 times with PBS, blocked in $3 \%$ bovine serum albumin (BSA) for $1 \mathrm{~h}$. Then cells were incubated with anti-FSP-1 antibody (1:500; cat. no. 07-2274; EMD Millipore, Billerica, CA, USA) and anti- $\alpha$-sarcomeric actin antibody (1:200; cat. no. A2547; Sigma-Aldrich, Merck KGaA) respectively, at $4^{\circ} \mathrm{C}$ overnight. Then, cells were washed twice, and incubated with corresponding secondary antibodies: Anti-rabbit IgG-FITC (1:200; cat. no. F1262; Sigma-Aldrich; Merck KGaA); and IgG-CY3 antibodies (1:400; cat. no. AP187C; Sigma-Aldrich; Merck $\mathrm{KGaA})$ at $37^{\circ} \mathrm{C}$ for $20 \mathrm{~min}$. DAPI $(1: 1,000$; cat. no. D8417; Sigma-Aldrich; Merck KGaA) was added to stain the nuclei. The positive cells were imaged using a confocal laser scanning microscope (Fluoview FV1000; Olympus Corporation, Tokyo, Japan).

Cell transfection. Small interfering RNA (siRNA) against NOD2 and NC siRNA were purchased from Ruibo Biotechnology Co., Ltd. (Guangzhou, China). The sequences used were as follows: Sense, 5'-AGAGAAAUUCUUUGA ATT-3', anti-sense 5'-UUCAAAGUUGAAUUUCUCUTG-3' for NOD2, and sense, 5'-UUCUCCGGAACGUGUCAC GUTT-3', anti-sense 5'-ACGUGACACGUUCGGAGAATT-3' for negative control sequence. The siRNAs were diluted with OPTI-MEM medium to achieve a final concentration of $100 \mathrm{nM}$. The cells were transfected with siRNA using Lipofectamine ${ }^{\circledR} 2000$ for 48 h, (Invitrogen; Thermo Fisher Scientific, Inc.) according to the manufacturer's protocol. Then, cells were stimulated with $20 \mathrm{mM} \mathrm{HG}$ for $48 \mathrm{~h}$ to be used for further experiments. Experiments were repeated three times.

Hemodynamic evaluation. Mice were anaesthetized with $10 \%$ sodium pentobarbital $(40 \mathrm{mg} / \mathrm{kg})$, and the common carotid artery of mice was separated away, then a plastic catheter containing $1 \%$ liquemine was inserted into the left ventricles from the right common carotid artery. The left ventricular end-diastolic pressure (LVEDP), left ventricular systolic pressure (LVSP), maximum rates of increased and decreased left ventricular pressure $( \pm \mathrm{dP} / \mathrm{dt} \max )$ were determined by the multimedia biological signal acquisition and analysis system (BD Biosciences, Franklin Lakes, NJ, USA).

Histology and immunohistochemistry. The heart tissues separated from the experimental groups of mice were fixed with $4 \%$ paraformaldehyde for $24 \mathrm{~h}$ at room temperature, embedded in paraffin using a paraffin embedding machine and sectioned ( $5 \mu \mathrm{m}$ thick). The sections were then deparaffinized, then rehydrated with graded ethanol at room temperature, and stained using hematoxylin for 3-5 min and eosin for 1-2 min (H\&E) and Masson's staining (Sigma-Aldrich; Merck KGaA) at room temperature in accordance with the manufacturer's protocol. For TUNEL staining of tissue, the sections were stained with a 
terminal deoxynucleotidyl transferase dUTP nick end labeling (TUNEL) kit (Boster Bioengineering Company, Wuhan, China) according to the manufacturer's protocol. 3.3 diaminobenzidine (DAB, Vector Laboratories, Inc., Burlingame, CA, USA) was used as the chromogenic substrate. Sections were then counterstained with $10 \%$ haematoxylin for $1 \mathrm{~min}$ (ProSciTech, Thuringowa, Australia). Immunostaining pictures were captured under a light microscope (Olympus BX51; Olympus Corporation, Tokyo, Japan).

TUNEL staining. Primary cardiomyocytes were transfected with NOD2 siRNA and siRNA NC, then stimulated with $20 \mathrm{mM} \mathrm{HG}$ for $48 \mathrm{~h}$. Cells were stained using fluorescein isothiocyanate (FITC)-labeled TUNEL for $1 \mathrm{~h}$ at $37^{\circ} \mathrm{C}$ following the protocol of the TUNEL fluorescent kit (Beyotime Institute of Biotechnology, Haimen, China). The TUNEL-positive cells were imaged under a laser scanning confocal microscope (Olympus Corporation) and five fields in each image were quantified (the number of green spots in each photograph).

ELISA. Angiontensin (Ang) II (Enzo Biochem, Inc., New York, NY, USA), cTn I and CK-MB (R\&D Systems, Minneapolis, MN, USA) in serum of mice, or TNF- $\alpha$ (cat. no. MTA00B), IL-1 $\beta$ (cat. no. MLB00C) and IL-6 (cat. no. DY406-05; R\&D Systems, Minneapolis, MN, USA) levels in the supernatants of cardiomyocytes were measured by ELISA kits following the manufacturer's protocol.

Assessment of cell proliferation. Proliferation of HG and NOD2 siRNA-treated CFs was evaluated using the Cell Counting Kit-8 (CCK-8; MultiSciences Biotechnology, Zhejiang, China) according to the manufacturer's protocol. Briefly, CFs were seeded onto 96-well plates for 12, 24, 36, 48 and $72 \mathrm{~h}$, then optical density (OD) was read at a wavelength of $450 \mathrm{~nm}$ with an automated microplate reader (Bio-Rad Laboratories, Inc., Hercules, CA, USA).

$R N A$ extraction and reverse transcription-quantitative polymerase chain reaction $(R T-q P C R)$. Total RNA was extracted from mouse cardiomyocytes and CFs using TRIzol ${ }^{\circledR}$ reagent (Invitrogen; Thermo Fisher Scientific, Inc.). Total RNA $(1 \mu \mathrm{g})$ was transcribed to cDNA with PrimeScript RT reagent kit (Takara Bio, Inc., Otsu, Japan) at $37^{\circ} \mathrm{C}, 15 \mathrm{~min}$ and $85^{\circ} \mathrm{C}, 5 \mathrm{sec}$. The sequences for primers were as follows: Forward, 5'-GCC TTCCTTCTACAGCACGT-3'; and reverse, 5'-TGGCAGGGC TCTTCTGCAAG-3 for NOD2; forward, 5'-GAATTGCTA TGTGTCTGGGT-3'; and reverse, 5'-CATCTTCAAACC TCCATGATG-3' for $\beta$-actin. The expression level of genes of interest was quantified using $20 \mu \mathrm{l}$ reaction mixture containing $1 \mu \mathrm{g}$ cDNA, $0.2 \mathrm{mmol} / 1$ of each primer and SYBR-Green (BioRad laboratories, Inc.) in 96-well plates in a LightCycler rapid thermal cycler system (MJ Research PTC-2000, MJ Research, Waltham, MA, USA). RT-qPCR amplification conditions were as follows: $95^{\circ} \mathrm{C}$ for $10 \mathrm{~min}, 40$ cycles at $95^{\circ} \mathrm{C}$ for $10 \mathrm{sec}$ and $60^{\circ} \mathrm{C}$ for $45 \mathrm{sec}$, and relative expression of genes were analyzed by the $2^{-\Delta \Delta \mathrm{Cq}}(24)$ calculation method and standardized against the housekeeping gene $\beta$-actin.

Western blotting. Protein was extracted from the heart tissues and cells using a protein extraction kit (BC3710; Beijing

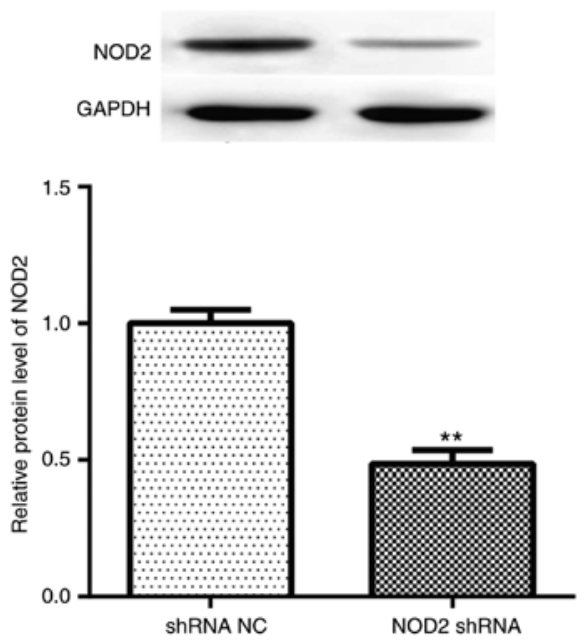

Figure 1. Efficiency of RNA interference. Expression of NOD2 protein detected by western blotting, following the injection of NOD2 shRNA in diabetic mice $(72 \mathrm{~h}) .{ }^{* *} \mathrm{P}<0.01$ vs. shRNA NC. NOD2, nucleotide-binding oligomerization domain 2; shRNA, short hairpin; NC, negative control.

Solarbio Science \& Technology Co., Ltd Solarbio, Beijing, China) and protein concentration was quantified using a BCA assay (Thermo Fisher Scientific, Inc.) according to the manufacturer's protocol. Protein $(20 \mu \mathrm{g})$ was separated by $10 \%$ sodium dodecyl sulfate-polyacrylamide gel electrophoresis (SDS-PAGE) and transferred onto a nitrocellulose membrane (EMD Millipore). Following blocking with 5\% nonfat milk in Tris buffered saline Tween-20, the blots were incubated with primary antibodies: Anti-NOD2 (1:500; sc-30080; Santa Cruz Biotechnology, Inc., Dallas, TX, USA), anti-collagen I (1:1,000; ab34710; Abcam, Cambridge, MA, USA), anti-collagen III (1:1,000; ab7778; Abcam), anti-TGF- $\beta$ (1:1,000; ab31013; Abcam) and GAPDH (1:5,000; sc-25778; Santa Cruz Biotechnology, Inc.) overnight at $4^{\circ} \mathrm{C}$. Then, the membranes were incubated with horseradish peroxidase-conjugated rabbit secondary antibodies (1:2,000; K5007; Dako, Agilent Technologies, Inc., Santa Clara, CA, USA) for $1 \mathrm{~h}$ at room temperature. The protein bands were visualized using the SuperSignal chemiluminescent detection module (34080; Pierce, Thermo Fisher Scientific, Inc.) and images were collected using a Tanon-5200 imaging system (Tanon Science and Technology Co., Ltd., Shanghai, China).

Statistical analysis. All statistical analysis was conducted using SPSS software, version 18.0 (SPSS, Inc., Chicago, IL, USA). One-way analysis of variance followed by the SNK or Dunnet's t test was used to evaluate the significance of differences in the data. The experiments were repeated three times and data are presented as the mean \pm standard error of the mean. $\mathrm{P}<0.05$ was considered to indicate a statistically significant difference.

\section{Results}

NOD2 inhibition ameliorates diabetes-induced myocardial apoptosis and fibrosis. To ascertain whether NOD2 plays a role in myocardial apoptosis and fibrosis associated with diabetes, the present study investigated the effect of NOD2 gene silencing 

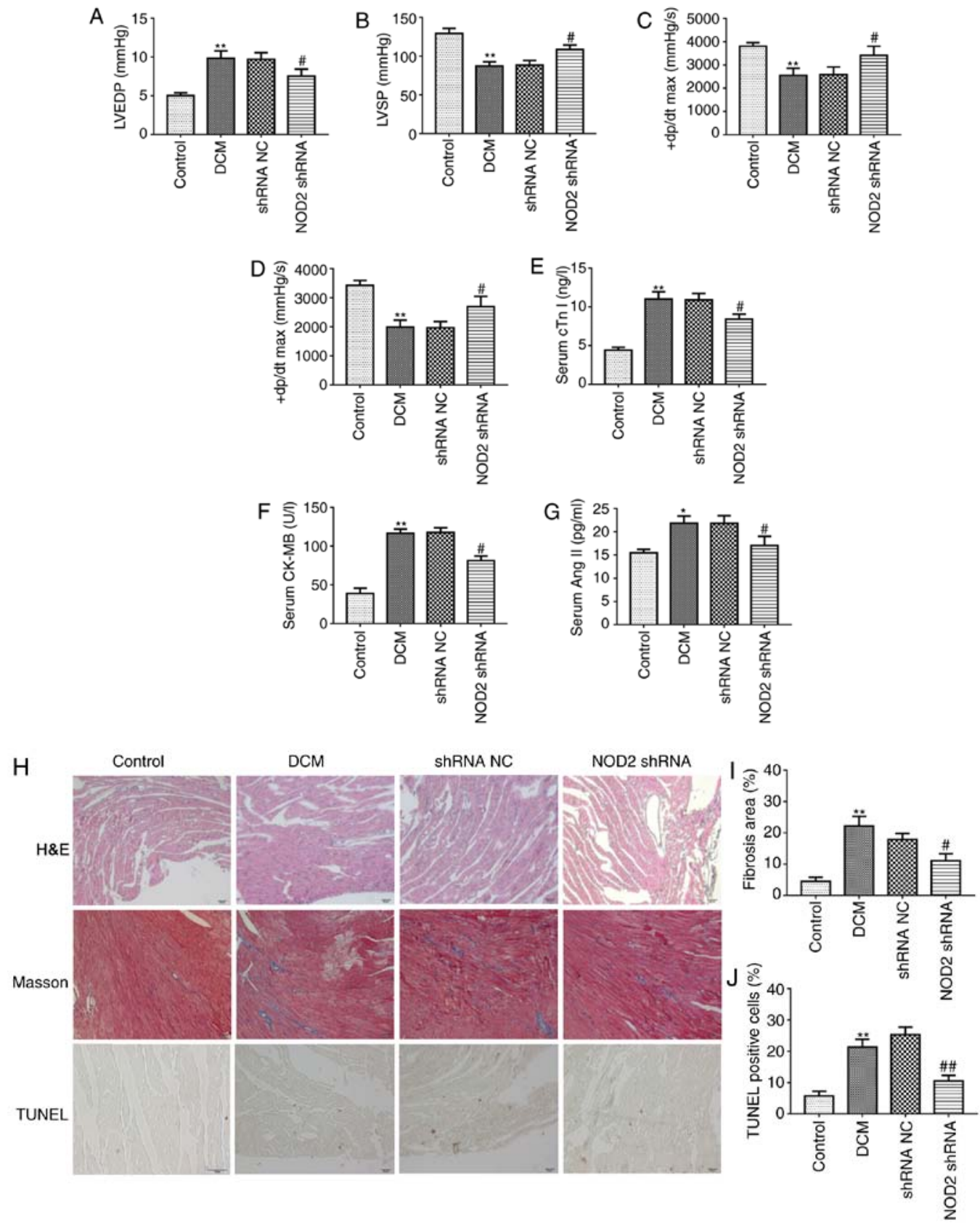

Figure 2. Pathological changes of the diabetic mouse model. Tests of cardiac function index in each group ( $\mathrm{n}=8)$. Measurements of (A) LVEDP, (B) LVSP, (C) $+\mathrm{dP} / \mathrm{dtmax}$ and (D) $-\mathrm{dP} / \mathrm{dtmax}$ in diabetic mice. Detection of the myocardial injury biomarkers in mouse serum (n=8). Measurement of (E) cTn I, (F) CK-MB, (G) Ang II. (H) Representative images of HE, Masson, TUNEL staining. Scale bars=20 $\mu \mathrm{m}$. Quantitative analysis of (I) myocardial fibrosis and (J) TUNEL-positive cells. ${ }^{* *} \mathrm{P}<0.01$ vs. control group, ${ }^{\#} \mathrm{P}<0.05,{ }^{\# \#} \mathrm{P}<0.01$ vs. shRNA NC group. TUNEL, terminal deoxynucleotidyl transferase dUTP nick end labeling; H\&E, hematoxylin and eosin; NOD2, nucleotide-binding oligomerization domain 2; shRNA, short hairpin; NC, negative control; LVEDP, left ventricular end-diastolic pressure; LVSP, left ventricular systolic pressure; $\pm \mathrm{dP} / \mathrm{dt}$ max, maximum rates of increased and decreased left ventricular pressure; DCM, diabetic cardiomyopathy.

in diabetic mice. STZ induced rapid hyperglycemia in mice compared with control at 1 week following injection. After the injection of NOD2 shRNA in diabetic mice (72 h), NOD2 shRNA treatment downregulated myocardial NOD2 protein levels compared with vehicle treatment (Fig. 1; P<0.01), which demonstrated that NOD2 shRNA lentivectors were successfully introduced into the mice. The hemodynamic indexes were first compared among the 4 groups at the end of the study. As compared with control group, the DCM model group exhibited a significant increase in LVEDP level (Fig. 2A; $\mathrm{P}<0.01$ ), however the LVSP and $\pm \mathrm{dP} / \mathrm{dt}$ max levels were markedly decreased
(Fig. 2B-D; P<0.01). In diabetic mice, LVEDP level was markedly reduced in NOD2 shRNA treatment group compared with shRNA NC group (Fig. 2A; P<0.05). However, NOD2 shRNA treatment upregulated LVSP, $+\mathrm{dP} / \mathrm{dt}$ max and $-\mathrm{dP} / \mathrm{dt}$ max levels compared with shRNA NC group (Fig. 2B-D; P<0.05). In addition, the data of the myocardial injury biomarkers revealed that cTn I, CK-MB and Ang II in serum from DCM model were significantly increased compared with control group. However, NOD2 gene elimination markedly reduced the levels of these factors in diabetic mice, compared with the effect exhibited by the shRNA NC treatment group (Fig. 2E-G; $\mathrm{P}<0.05$ ). 

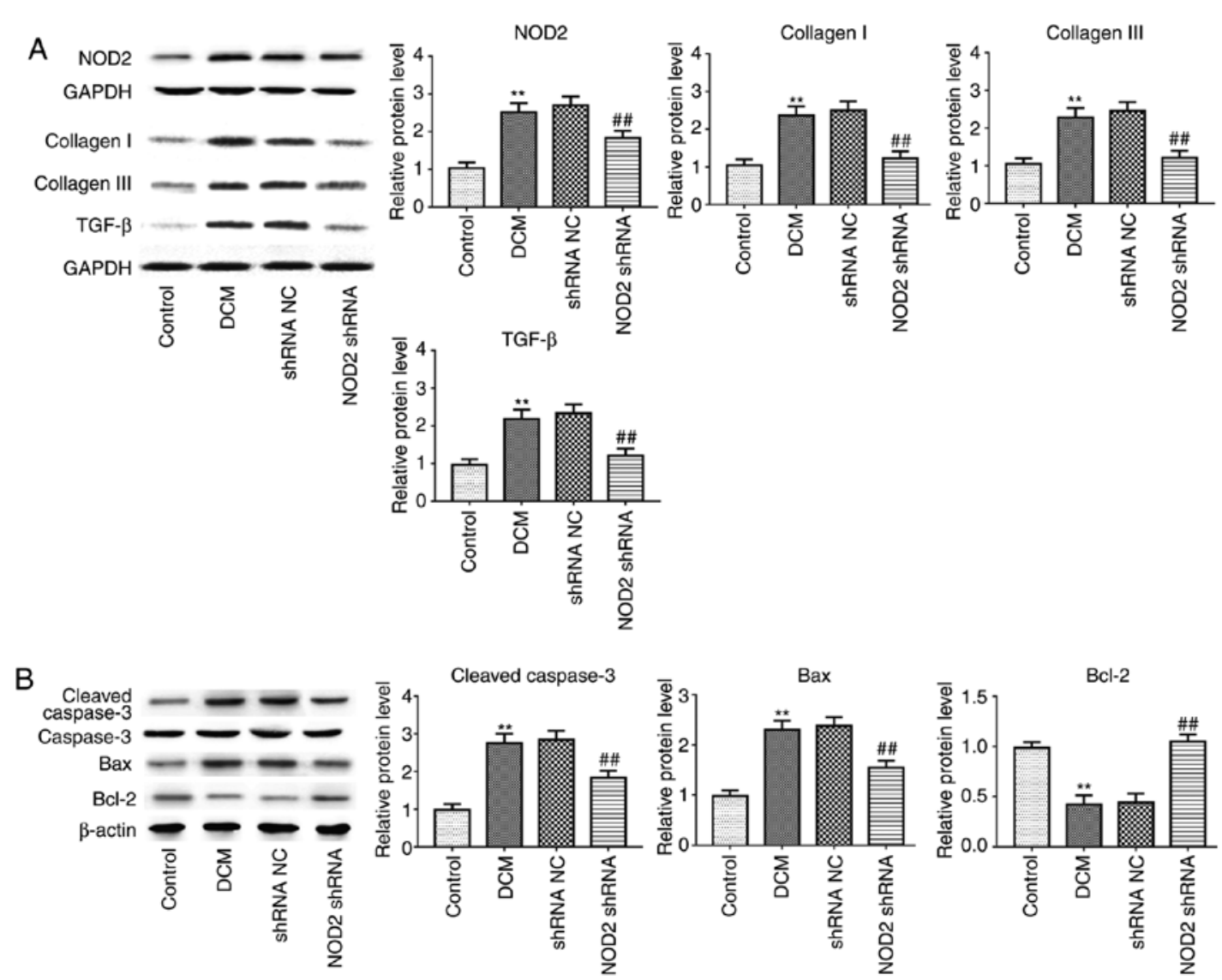

Figure 3. Suppressing NOD2 with NOD2 shRNA attenuates collagen and apoptosis-related protein expression in diabetic mice. (A) Western blot analysis of protein expression of NOD2, collagen I, collagen III and TGF- $\beta 1$. (B) Western blot analysis of protein expression of Caspase-3, Bax and Bcl-2. ${ }^{* *} \mathrm{P}<0.01$ vs. control group, ${ }^{\# \#} \mathrm{P}<0.01$ vs. shRNA NC group. NOD2, nucleotide-binding oligomerization domain 2; shRNA, short hairpin; NC, negative control; DCM, diabetic cardiomyopathy; TGF- $\beta$, transforming growth factor- $\beta$; Bcl-2, B cell lymphoma 2 ; Bax, Bcl-2 associated X, apoptosis regulator.

Heart tissues were isolated from the experimental mice, and different staining methods were used to detect the effect of NOD2 shRNA on pathological changes in diabetic mice. As presented in Fig. $2 \mathrm{H}$, The pathological changes by H\&E stain in control group showed that myocardial cells were orderly arranged and the nuclei were homogeneous. However, in the DCM group, the myocardium exhibited a disordered arrangement, the nucleus was not uniform and part of myocardial fiber was fractured. The NOD2 shRNA treatment lessened injury of heart tissue in diabetic mice. Masson's staining of heart sections revealed more ECM in the interstitial regions of the DCM group than myocardium of control group, and apoptosis in cardiac tissue was assayed using TUNEL staining. Quantitative analysis of Masson's staining revealed a significant increase in collagen deposition of DCM mice compared with control (Fig. 2I; P<0.01). However, NOD2 shRNA treatment ameliorated collagen deposition (Fig. 2I; $\mathrm{P}<0.05$ vs. shRNA NC). Quantitative analysis of TUNEL stain revealed an increased number of TUNEL-positive cells in the DCM group (Fig. 2J; $\mathrm{P}<0.01$ vs. control group). Compared to the vehicle treatment group, NOD2 silencing weakened cell apoptosis (Fig. 2J; $\mathrm{P}<0.01$ vs. shRNA NC group).

Furthermore, in line with alteration of collagen deposition and apoptosis detection in immunostaining, collagen I, collagen III, TGF- $\beta 1$ (Fig. $3 \mathrm{~A} ; \mathrm{P}<0.01$ ), and apoptosis-related proteins Caspase- 3 and $\mathrm{B}$ cell lymphoma (Bcl)-2 associated $\mathrm{X}$, apoptosis regulator (Bax) expression levels were significantly increased in the DCM group, as demonstrated by western blotting, but the expression of these proteins was attenuated in diabetic mice with NOD2 shRNA treatment, compared with vehicle-treated mice. Suppression of NOD2 significantly upregulated Bcl-2 expression, and downregulated NOD2 expression in diabetic mice (Figs. $3 \mathrm{~A}$ and $2 \mathrm{~B} ; \mathrm{P}<0.01$ ).

NOD2 is upregulated in HG-induced primary cardiomyocytes and CFs. Immunofluorescence staining was performed to identify primary cardiomyocytes and CFs. The results demonstrated that staining of $\alpha$-sarcomeric actin and FSP-1 were positive (Fig. 4), reflecting the isolated cells were primary cardiomyocytes and CFs. The expression of NOD2 mRNA and protein in primary cells exposed to glucose for $48 \mathrm{~h}$ was further detected by RT-qPCR and western blotting. The results revealed that NOD2 levels in primary cardiomyocytes were significantly increased at concentrations of 15,20 and $25 \mathrm{mM}$ glucose (Fig. 4B and C; $\mathrm{P}<0.05$ vs. control group). Similarly, NOD2 level in CFs was enhanced at concentrations of 15 , 20 and $25 \mathrm{mM}$ glucose treatment (Fig. 4E and F; P<0.01 vs. control group).

Suppressing NOD2 attenuates inflammatory factor expression and apoptosis in cardiomyocytes. Myocardial cell apoptosis and myocardial fibrosis are the primary pathological changes in diabetic cardiomyopathy (5). Given this, the present study explored the effect of NOD2 silencing on cardiomyocyte 

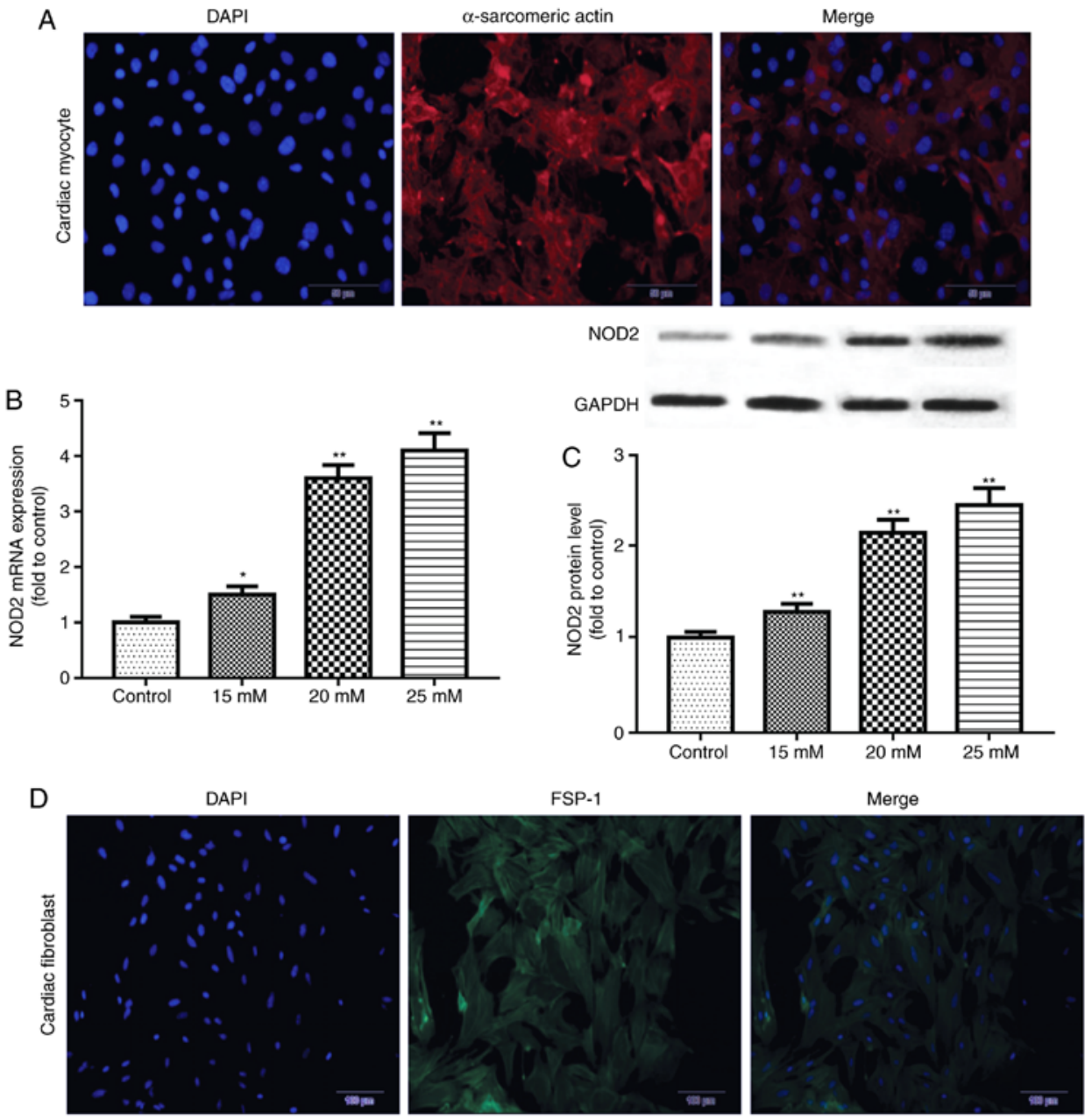

F

NOD2
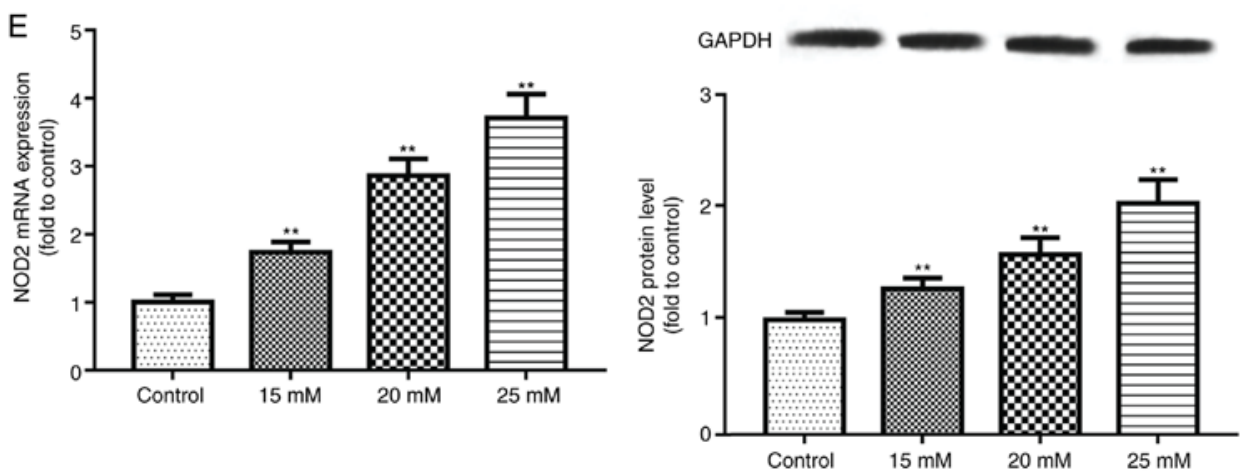

Figure 4. Identification of primary cardiomyocytes and cardiac fibroblasts. (A) Immunostaining of $\alpha$-sarcomeric actin in primary cardiomyocytes. Scale bars $=50 \mu \mathrm{m}$. (B) RT-qPCR analysis of NOD2 mRNA expression in primary cardiomyocytes stimulated with different concentrations of high glucose. (C) Western blot analysis of NOD2 protein level in primary cardiomyocytes stimulated with different concentrations of high glucose. (D) Immunostaining of FSP-1. Scale bars $=100 \mu \mathrm{m}$. (E) RT-qPCR analysis of NOD2 mRNA expression in CFs stimulated with different concentrations of high glucose. (F) Western blot analysis of NOD2 protein level in CFs stimulated with different concentrations of high glucose. ${ }^{*} \mathrm{P}<0.05,{ }^{* *} \mathrm{P}<0.01$ vs. control group. NOD2, nucleotide-binding oligomerization domain 2; CFs, cardiac fibroblasts; RT-qPCR, reverse transcription-quantitative polymerase chain reaction.

apoptosis following $20 \mathrm{mM} \mathrm{HG}$ treatment for $48 \mathrm{~h}$. The interference efficiency of NOD2 using siRNA in primary cardiomyocytes was evaluated by western blotting. The result revealed that treatment with NOD2 siRNA had a marked inhibition on NOD2 protein expression (Fig. 5A; P<0.01), reflecting transfection efficiency of interfering RNA. It has been reported that cardiomyocyte apoptosis is associated with inflammatory factors (25). Next, ELISA was used to assess the cytokine levels in different groups. As presented in Fig. 5B, the expression levels of TNF- $\alpha$, IL-1 $\beta$ and IL- 6 in HG-stimulated cardiomyocytes were significantly increased compared with control group $(\mathrm{P}<0.01)$. Furthermore, compared with the siRNA NC group, NOD2 silencing significantly suppressed TNF- $\alpha$, IL-1 $\beta$ and IL-6 expression levels (Fig. 5B; P<0.05). In addition, the present study detected the apoptosis of cardiomyocytes using a TUNEL assay. The results revealed that 


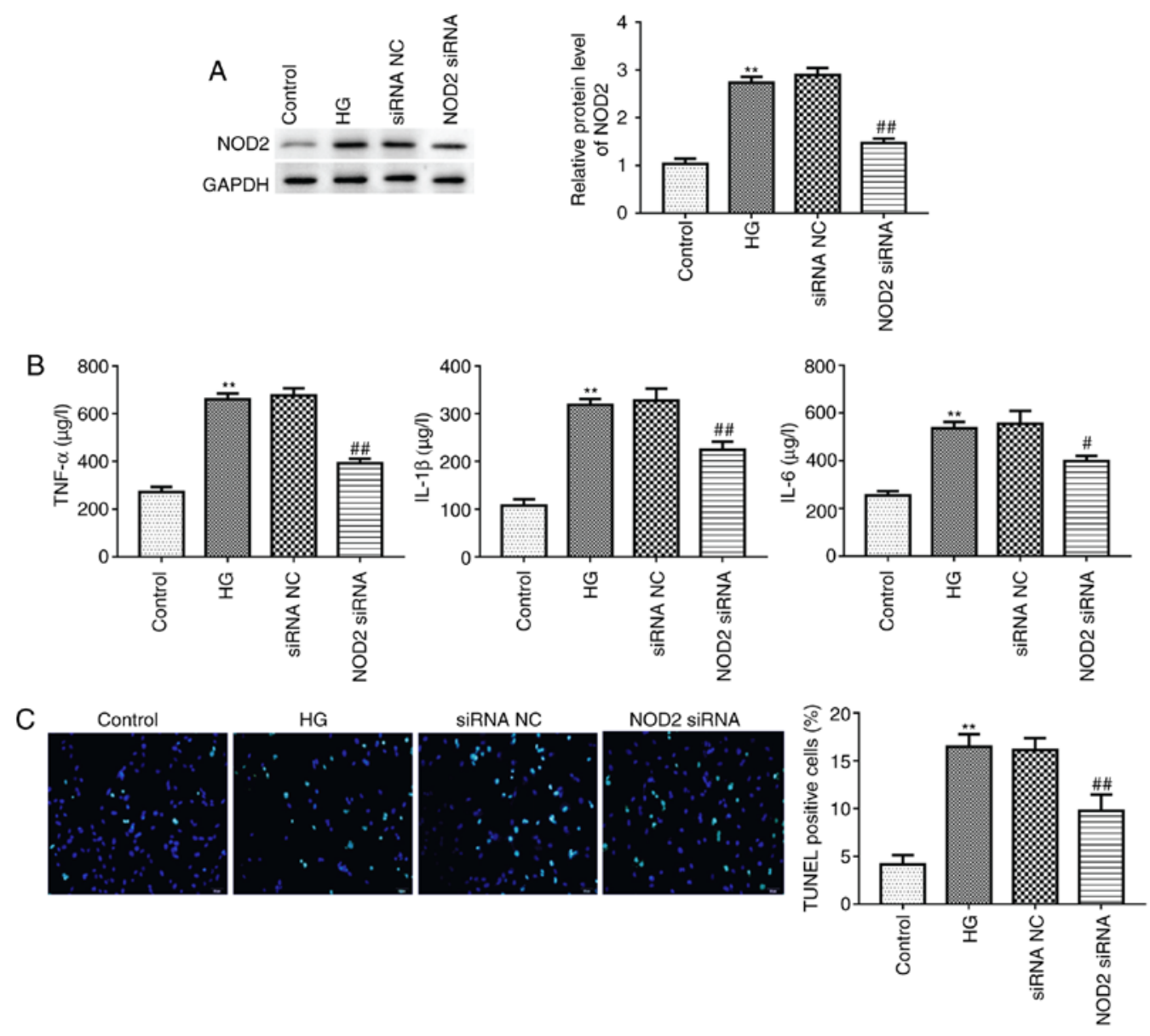

Figure 5. Effect of NOD2 silencing on cardiomyocyte apoptosis. (A) The expression of NOD2 in different groups. (B) The expression levels of TNF- $\alpha$, IL-1 $\beta$ and IL-6 in different groups. (C) NOD2 silencing effect on apoptosis of cardiomyocytes. Scale bars $=50 \mu \mathrm{m}$. "* $\mathrm{P}<0.01$ vs. control group; ${ }^{*} \mathrm{P}<0.05,{ }^{\# \#} \mathrm{P}<0.01 \mathrm{vs}$. siRNA NC group. NOD2, nucleotide-binding oligomerization domain 2; si, small interfering; NC, negative control; HG, high glucose; TNF- $\alpha$, tumor necrosis factor- $\alpha$; IL, interleukin.

there were more TUNEL-positive cells in HG treatment group compared with control group (Fig. 5C; P<0.01). The NOD2 siRNA group revealed decreased green fluorescence compared with the siRNA NC group (Fig. 5C; $\mathrm{P}<0.01$ ), indicating that knockdown of NOD2 inhibited HG-induced cardiomyocyte apoptosis.

NOD2 silencing suppresses cell proliferation and myocardial fibrosis. Next, the present study detected the effect of NOD2 silencing on fibrosis of CFs with $48 \mathrm{~h} \mathrm{HG}$ incubation in vitro. As presented in Fig. 6A, the proliferation of CFs was significantly increased in the HG-treated group compared with control group, as detected by CCK-8 assay $(\mathrm{P}<0.01)$. However, compared to siRNA NC group, the cell proliferation in NOD2 siRNA group was significantly decreased (Fig. 6A; $\mathrm{P}<0.05)$. In addition, the results of western blotting revealed that the expression of NOD2, collagen I, collagen III, TGF- $\beta$, Caspase-3 and Bax proteins were upregulated in HG group compared with control group, whereas as compared with siRNA NC group, the expression levels of these proteins in the NOD2 siRNA group were downregulated. Conversely, Bcl-2 expression levels exhibited the opposite trends. The aforementioned results suggested that NOD2 silencing suppressed collagen production in $\mathrm{HG}$-induced $\mathrm{CFs}$ and decreased myocardial cell apoptosis (Fig. 6B and $\mathrm{C} ; \mathrm{P}<0.01$ ).

\section{Discussion}

DCM is associated with diabetes-induced changes in cardiac structure and function, and is a disorder characterized by consistent diastolic dysfunction and increased ventricular mass $(22,26)$. DCM is closely associated with high incidence and high mortality rates of heart failure in diabetic patients (27). The possible mechanisms for the development of DCM are myocardial metabolic disorders and myocardial fibrosis, inflammation, myocardial apoptosis and autophagy, resulting in left ventricular hypertrophy, diastolic and systolic dysfunction, which ultimately form congestive heart failure (18,28-30). DCM results from a variety of mechanisms and signaling pathways in myocardial cells in the high glucose state $(31,32)$.

The present study revealed that in the DCM model, LVEDP was significantly increased compared with control, but the LVSP, $+\mathrm{dP} / \mathrm{dt}$ max and $-\mathrm{dP} / \mathrm{dt}$ max levels were markedly decreased. However, NOD2 shRNA treatment reversed these results. In addition, the results for pathological changes in diabetic mice revealed that collagen deposition in DCM mice was significantly increased compared with control. However, NOD2 silencing ameliorated collagen deposition in the heart tissue of diabetic mice. Furthermore, TUNEL staining indicated that the DCM group had an increased number of TUNEL-positive cells compared with control group, and 

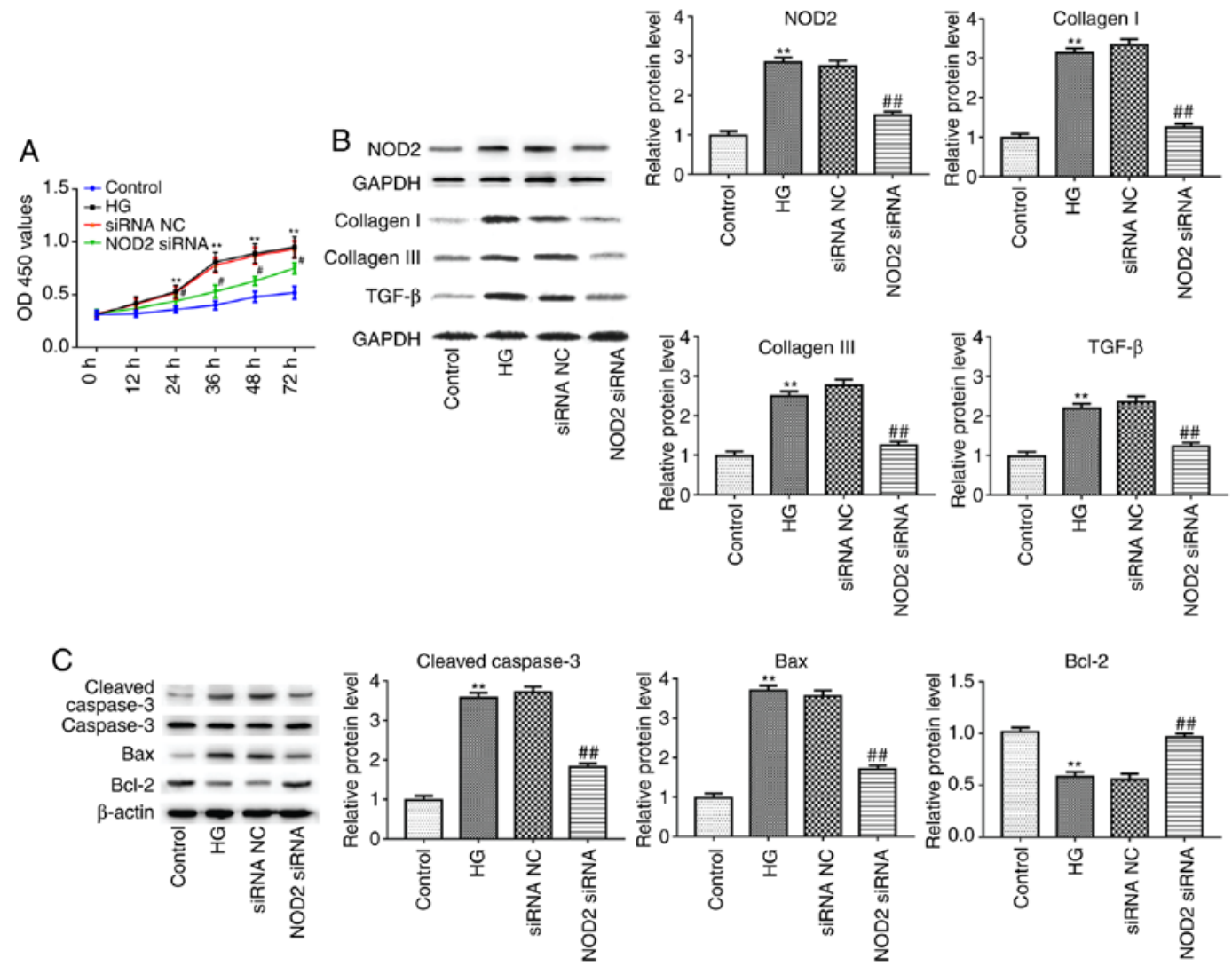

Figure 6. Effect of NOD2 silencing on cardiac fibroblasts. (A) NOD2 silencing reduced proliferation of cardiac fibroblasts. (B) The protein expression levels of NOD2, collagen I, collagen III and TGF- $\beta$ in cardiac fibroblasts in different groups. (C) The protein expression levels of Caspase-3, Bax and Bcl-2 from cardiomyocytes in different groups. ${ }^{* *} \mathrm{P}<0.01$ vs. control group; ${ }^{*} \mathrm{P}<0.05,{ }^{\# \#} \mathrm{P}<0.05$ vs. siRNA NC group. NOD2, nucleotide-binding oligomerization domain 2 ; si, small interfering; NC, negative control; HG, high glucose; TGF- $\beta$, transforming growth factor- $\beta$; Bcl-2, B cell lymphoma 2 ; Bax, Bcl-2 associated X, apoptosis regulator; OD, optical density.

NOD2 silencing weakened cell apoptosis. Expression levels of collagen I, collagen III and TGF- $\beta 1$ proteins were significantly increased in the DCM group compared with control, but the expression of these proteins in NOD2 shRNA treated mice were lower compared with in vehicle-treated mice. These findings suggested that NOD2 silencing ameliorated diabetes-induced myocardial apoptosis and fibrosis.

The NOD-like receptor family is composed of more than 20 members, each of which consist of three distinct domains. Of these, the N-terminal effect domains include caspase recruitment region, pyrin region or apoptotic repeat, and the most representative is NOD2 $(33,34)$. It has previously been demonstrated that NOD2 is a class of endogenous substance subjected to high blood sugar, high blood lipids, oxidants and inflammatory cell infiltration, leading to persistent tissue injury in the inflammatory state (35). It is reported that NOD2 promotes the expression of nuclear factor- $\kappa \mathrm{B}, \mathrm{TNF}-\alpha, \mathrm{IL}-1 \beta$ and other inflammatory factors (20). Knockdown of NOD2 results in reduced renal damage compared with wild type C57BL/6 mice (36). In HG-induced cardiomyocytes cells, it was demonstrated that TNF- $\alpha$, IL- $1 \beta$ and IL- 6 levels in HG-stimulated cardiomyocytes were significantly increased compared with in the control group. However, NOD2 silencing suppressed TNF- $\alpha$, IL-1 $\beta$ and IL- 6 expression and inhibited cell apoptosis. These data indicated that knockdown of NOD2 inhibited HG-induced inflammatory factor expression and cardiomyocyte apoptosis.
Activation of fibroblasts leads to myocardium fibrosis (37). TGF- $\beta$, a class of complex growth factor, has five subunits $\beta 1-\beta 5$, and TGF- $\beta 1$ is a major regulator in the mechanism of myocardial fibrosis and affects the synthesis of collagen fibers through a series of cascade reactions (38). In the established DCM mouse model, NOD2, collagen I, collagen III and TGF- $\beta$ protein expression levels were significantly increased. However, NOD2 silencing reduced NOD2, collagen I, collagen III and TGF- $\beta$ protein expression. In addition, the proliferation of CFs was significantly increased in HG-treated cells compared with the normal group, but compared with the siRNA NC group, the cell proliferation in the NOD2 siRNA group was significantly decreased. These results suggested that NOD2 silencing inhibited cell proliferation and myocardial fibrosis.

In conclusion, the present study investigated the role and mechanism of the NOD-like receptor NOD2 in the pathogenesis of DCM. The results provide direct evidence that NOD2 plays a role in the process of hyperglycemia-induced cardiomyocyte apoptosis and cardiac fibrosis. A novel effective target NOD2 for the prevention and treatment of DCM has been screened, which has an important theoretical significance and potential value for clinical application.

\section{Acknowledgements}

Not applicable. 


\section{Funding}

The present study was supported by Shandong Provincial Natural Science Foundation, China (grant no.ZR2015HM052); key research and development plan of Shandong Province (grant no. 2017GSF218012) and China Postdoctoral Science Foundation (grant no. 2014M561936).

\section{Availability of data and materials}

The datasets used and/or analyzed during the current study are available from the corresponding author on reasonable request.

\section{Authors' contributions}

Guarantor of integrity of the entire study: YH; study concepts: LS, YH; study design: LS, YH, LL; definition of intellectual content: LS, ML; analysis of pathology: ML, WY; literature research: WW, WY; clinical studies: LS, WW, WY; experimental studies: LS, YH; data acquisition: LL, WL; statistical analysis: LS; manuscript editing: LS, LL, ML; manuscript reviewing: LS, LL.

\section{Ethics approval and consent to participate}

All experimental protocols were approved by the Institutional Animal Care and Use Committee of Shandong University (Jinan, China).

\section{Patient consent for publication}

Not applicable.

\section{Competing interests}

The authors declare that they have no competing interests.

\section{References}

1. Hillis GS, Woodward M, Rodgers A, Chow CK, Li Q, Zoungas S, Patel A, Webster R, Batty GD, Ninomiya T, et al: Resting heart rate and the risk of death and cardiovascular complications in patients with type 2 diabetes mellitus. Diabetologia 55: 1283-1290, 2012.

2. Adameova A and Dhalla NS: Role of microangiopathy in diabetic cardiomyopathy. Heart Fail Rev 19: 25-33, 2014.

3. Bielecka-Dabrowa A, Wierzbicka M, Dabrowa M and Goch A: New methods in laboratory diagnostics of dilated cardiomyopathy. Cardiol J 15: 388-395, 2008.

4. Carneiro LA, Magalhaes JG, Tattoli I, Philpott DJ and Travassos LH: Nod-like proteins in inflammation and disease. J Pathol 214: 136-148, 2008.

5. Zhang J, Li B, Zheng Z, Kang T, Zeng M, Liu Y and Xia B: Protective effects of Notch1 signaling activation against high glucose-induced myocardial cell injury: Analysis of its mechanisms of action. Int J Mol Med 36: 897-903, 2015.

6. DeMarco VG, Aroor AR and Sowers JR: The pathophysiology of hypertension in patients with obesity. Nat Rev Endocrinol 10: 364-376, 2014.

7. Lorenzo O, Picatoste B, Arescarrasco S, Ramírez E, Egido J and Tuñón J: Potential role of nuclear factor $\kappa \mathrm{B}$ in diabetic cardiomyopathy. Mediators Inflamm 2011: 652097, 2011.

8. Zhang B, Qiang S, Chen Y, Pan R, Kuang S, Liu G, Sun G and Sun X: Myricitrin alleviates oxidative stress-induced inflammation and apoptosis and protects mice against diabetic cardiomyopathy. Sci Rep 7: 44239, 2017.
9. Mohammadshahi M, Haidari F and Soufi FG: Chronic resveratrol administration improves diabetic cardiomyopathy in part by reducing oxidative stress. Cardiol J 21: 39-46, 2014

10. Qin WD, Liu GL, Wang J, Wang H, Zhang JN, Zhang F, Ma Y, Ji XY, Li C and Zhang MX: Poly(ADP-ribose) polymerase 1 inhibition protects cardiomyocytes from inflammation and apoptosis in diabetic cardiomyopathy. Oncotarget 7: 35618-35631, 2016.

11. Song JQ, Teng X, Cai Y, Tang CS and Qi YF: Activation of Akt/GSK-3beta signaling pathway is involved in intermedin(1-53) protection against myocardial apoptosis induced by ischemia/reperfusion. Apoptosis 14: 1299-1307, 2009.

12. Ares-Carrasco S, Picatoste B, Benito-Martín A, Zubiri I, Sanz AB, Sánchez-Niño MD, Ortiz A, Egido J, Tuñón J and Lorenzo O: Myocardial fibrosis and apoptosis, but not inflammation, are present in long-term experimental diabetes. Am J Physiol Heart Circ Physiol 297: H2109-H2119, 2009.

13. Vadla GP and Vellaichamy E: Anti-fibrotic cardio protective efficacy of aminoguanidine against streptozotocin induced cardiac fibrosis and high glucose induced collagen up regulation in cardiac fibroblasts. Chem Biol Interact 197: 119-128, 2012.

14. Wang HX, Zhang QF, Zeng XJ, Wang W, Tang CS and Zhang LK: Effects of angiotensin III on protein, DNA, and collagen synthesis of neonatal cardiomyocytes and cardiac fibroblasts in vitro. J Cardiovasc Pharmacol Ther 15: 393-402, 2010.

15. Wang WK, Wang B, Lu QH, Zhang W, Qin WD, Liu XJ, Liu XQ, An FS, Zhang Y and Zhang MX: Inhibition of high-mobility group box 1 improves myocardial fibrosis and dysfunction in diabetic cardiomyopathy. Int J Cardiol 172: 202-212, 2014.

16. Westermann D, Rutschow S, Jäger S, Linderer A, Anker S, Riad A, Unger T, Schultheiss HP, Pauschinger M and Tschöpe C: Contributions of inflammation and cardiac matrix metalloproteinase activity to cardiac failure in diabetic cardiomyopathy: The role of angiotensin type 1 receptor antagonism. Diabetes 56: 641-646, 2007.

17. Correa RG, Milutinovic S and Reed JC: Roles of NOD1 (NLRC1) and NOD2 (NLRC2) in innate immunity and inflammatory diseases. Biosci Rep 32: 597-608, 2012.

18. Li CJ, Lv L, Li H and Yu DM: Cardiac fibrosis and dysfunction in experimental diabetic cardiomyopathy are ameliorated by alpha-lipoic acid. Cardiovasc Diabetol 11: 73, 2012.

19. Geddes K, Magalhães JG and Girardin SE: Unleashing the therapeutic potential of NOD-like receptors. Nat Rev Drug Discov 8: 465-479, 2009

20. Liu Y, Yang H, Liu LX, Yan W, Guo HJ, Li WJ, Tian C, Li HH and Wang HX: NOD2 contributes to myocardial ischemia/reperfusion injury by regulating cardiomyocyte apoptosis and inflammation. Life Sci 149: 10-17, 2016.

21. Perez-Chanona E, Mühlbauer M and Jobin C: The microbiota protects against ischemia/reperfusion-induced intestinal injury through nucleotide-binding oligomerization domain-containing protein 2 (NOD2) signaling. Am J Pathol 184: 2965-2975, 2014.

22. Liu HQ, Zhang XY, Edfeldt K, Nijhuis MO, Idborg H, Bäck M, Roy J, Hedin U, Jakobsson PJ, Laman JD, et al: NOD2-mediated innate immune signaling regulates the eicosanoids in atherosclerosis. Arterioscler Thromb Vasc Biol 33: 2193-2201, 2013.

23. Du P, Fan B, Han H, Zhen J, Shang J, Wang X, Li X, Shi W, Tang W, Bao C, et al: NOD2 promotes renal injury by exacerbating inflammation and podocyte insulin resistance in diabetic nephropathy. Kidney Int 84: 265-276, 2013.

24. Livak KJ and Schmittgen TD: Analysis of relative gene expression data using real-time quantitative PCR and the 2(-Delta Delta C(T)) method. Methods 25: 402-408, 2001.

25. Vakhrusheva O, Smolka C, Gajawada P, Kostin S, Boettger T, Kubin T, Braun T and Bober E: Sirt7 increases stress resistance of cardiomyocytes and prevents apoptosis and inflammatory cardiomyopathy in mice. Circ Res 102: 703-710, 2008.

26. Falcão-Pires I and Leite-Moreira AF: Diabetic cardiomyopathy: Understanding the molecular and cellular basis to progress in diagnosis and treatment. Heart Fail Rev 17: 325-344, 2012.

27. Li X, Li F, Chu Y, Wang X, Zhang H, Hu Y, Zhang Y, Wang Z, Wei X, Jian W, et al: NOD2 deficiency protects against cardiac remodeling after myocardial infarction in mice. Cell Physiol Biochem 32: 1857-1866, 2013.

28. Zhao Y, Zhang L, Qiao Y, Zhou X, Wu G, Wang L, Peng Y, Dong X, Huang H, Si L, et al: Heme oxygenase-1 prevents cardiac dysfunction in streptozotocin-diabetic mice by reducing inflammation, oxidative stress, apoptosis and enhancing autophagy. PLoS One 8: e75927, 2013. 
29. Mano Y, Anzai T, Kaneko H, Nagatomo Y, Nagai T, Anzai A, Maekawa Y, Takahashi T, Meguro T, Yoshikawa T and Fukuda K: Overexpression of human C-reactive protein exacerbates left ventricular remodeling in diabetic cardiomyopathy. Circ J 75: 1717-1727, 2011

30. Liu ZW, Wang JK, Qiu C, Guan GC, Liu XH, Li SJ and Deng ZR: Matrine pretreatment improves cardiac function in rats with diabetic cardiomyopathy via suppressing ROS/TLR-4 signaling pathway. Acta Pharmacol Sin 36: 323-333, 2015.

31. Pan Y, Wang Y, Zhao Y, Peng K, Li W, Wang Y, Zhang J, Zhou S, Liu Q, Li X, et al: Inhibition of JNK phosphorylation by a novel curcumin analog prevents high glucose-induced inflammation and apoptosis in cardiomyocytes and the development of diabetic cardiomyopathy. Diabetes 63: 3497-3511, 2014.

32. Rubler S, Dlugash J, Yuceoglu YZ, Kumral T, Branwood AW and Grishman A: New type of cardiomyopathy associated with diabetic glomerulosclerosis. Am J Cardiol 30: 595-602, 1972.

33. Santos VN, Leite-Mór MM, Kondo M, Martins JR, Nader H, Lanzoni VP and Parise ER: Serum laminin, type IV collagen and hyaluronan as fibrosis markers in non-alcoholic fatty liver disease. Braz J Med Biol Res 38: 747-753, 2005.

34. Miki T, Yuda S, Kouzu H and Miura T: Diabetic cardiomyopathy: Pathophysiology and clinical features. Heart Fail Rev 18 149-166, 2013
35. Teshima Y, Takahashi N, Nishio S, Saito S, Kondo H, Fukui A, Aoki K, Yufu K, Nakagawa M and Saikawa T: Production of reactive oxygen species in the diabetic heart. Roles of mitochondria and NADPH oxidase. Circ J 78: 300-306, 2014.

36. Werner S, Krieg T and Smola H: Keratinocyte-fibroblast interactions in wound healing. J Invest Dermatol 127: 998-1008, 2007.

37. Zhang X, Ma X, Zhao M, Zhang B, Chi J, Liu W, Chen W, Fu Y, Liu Y and Yin X: $\mathrm{H} 2$ and $\mathrm{H} 3$ relaxin inhibit high glucose-induced apoptosis in neonatal rat ventricular myocytes. Biochimie 108: 59-67, 2015.

38. Zhang YC, Mou YL and Xie YY: Research progress in relations between renin angiotensin system and diabetic cardiomyopathy. Sheng Li Ke Xue Jin Zhan 42: 269-275, 2011 (In Chinese).

This work is licensed under a Creative Commons Attribution-NonCommercial-NoDerivatives 4.0 International (CC BY-NC-ND 4.0) License. 